\title{
MODELAMIENTO DEL CRECIMIENTO DE TORNILLO Cedrelinga catenaeformis Ducke EN PLANTACIONES EN JENARO HERRERA, DEPARTAMENTO DE LORETO, PERÚ
}

\author{
Juan B. BALUARTE VASQUEZ ${ }^{1}$, J.G. ALVAREZ GONZALEZ ${ }^{2}$ \\ 1 Consultor Independiente. Urb. César Calvo de Araujo A-10, San Juan Bautista, Loreto, Perú. E-mail: \\ jbaluarte@hotmail.com \\ 2 Escuela Politécnica Superior de Lugo, Universidad de Santiago de Compostela. 27002. Lugo, España. E-mail: \\ juangabriel.alvarez@usc.es
}

\section{RESUMEN}

Se procesaron los datos de crecimiento de 1.094 árboles de Cedrelinga catenaeformis para generar modelos de calidad de sitio y de crecimiento en diámetro, altura y volumen de rodal. Se emplearon ecuaciones en diferencias algebraicas derivadas de los modelos base de Hossfeld, Bertalanffy-Richards y Korf. En lo referente a la calidad de sitio, el modelo de Korf explicó más del 95\% de la variabilidad observada, asignando el $57 \%$ de las 23 parcelas forestales estudiadas a una calidad de sitio alta. La calidad del sitio fue incluida para ajustar los modelos de crecimiento en diámetro y altura del árbol y del volumen de rodal. El modelo de Bertalanffy-Richards mostró buenos resultados en diámetro, explicando algo más del $80 \%$ de la variabilidad observada; mientras que en altura, el modelo de Korf explicó más del 91\% de la variabilidad observada. En volumen de rodal, el modelo de Korf mostró los mejores resultados, con un porcentaje de variabilidad de más del $90 \%$. Según este modelo, las plantaciones de C. catenaeformis alcanzan volúmenes de 360 y $368 \mathrm{~m}^{3} \mathrm{ha}^{-1}$ a los 9 y 18 años en las clases de sitio alto y medio, respectivamente. El incremento corriente anual máximo en volumen culmina muy temprano, a los 3 años, alcanzando $36{\text { y } 56 \mathrm{~m}^{3} \mathrm{ha}^{-1} \text { año }}^{-1}$ en las clases de sitio medio y alto, respectivamente. El turno de rotación silvicultural se produce a los 9,7 y 5 años con crecimientos de 13 , 28 y $44 \mathrm{~m}^{3} \mathrm{ha}^{-1}$ año ${ }^{-1}$, en las clases de sitio bajo, medio y alto, respectivamente.

PALABRAS CLAVE: Plantaciones, Cedrelinga catenaeformis, índice de sitio, crecimiento, modelización forestal.

\section{MODELLING GROWTH DEVELOPMENT OF TORNILLO Cedrelinga catenaeformis Ducke PLANTATIONS IN LORETO DEPARTMENT, PERU}

\begin{abstract}
Growth data from 1094 trees of Cedrelinga catenaeformis planted in Jenaro Herrera were used to develop models of site quality, diameter and height individual-tree growth and stand volume growth. Algebraic difference equations derived from the base models of Hossfeld, Bertalanffy-Richards and Korf were used. Regarding the site quality model, the base model of Korf explained over $95 \%$ of the observed variability, and the $57 \%$ of the 23 studied forestry plots studied were classified as high quality sites. Site quality was included to adjust the fit of individual-tree and stand volume growth models. The base model of Bertalanffy-Richards showed the best results in individual-tree diameter growth, explaining more than $80 \%$ of the observed variability; while Korf model explained over $91 \%$ of the observed variability in individual-tree height growth. The base model of Korf showed the best results for stand volume growth, with a percentage of variability explained over $90 \%$. According to this model, the plantations of C. catenaeformis will reach volumes of 360 and $368 \mathrm{~m}^{3} \mathrm{ha}^{-1}$ at 9 and 18 years old for high and medium site qualities, respectively. The maximum current annual increment will be reached at 3 years old, with values of 36 and $56 \mathrm{~m}^{3} \mathrm{ha}^{-1} \mathrm{year}^{-1}$ in medium and high site qualities; while the rotation age corresponds to 9,7 and 5 years old, with values of 13,28 and $44 \mathrm{~m}^{3} \mathrm{ha}^{-1}$ year $^{-1}$, for low, medium and high site qualities, respectively.
\end{abstract}

KEYWORDS: Forestry plantations, Cedrelinga catenaeformis, site index, growth, forest modelling. 


\section{INTRODUCCIÓN}

Cedrelinga catenaeformis Ducke "tornillo" de la familia Fabaceae, es una especie maderable muy promisoria para la reforestación en la Amazonía. Otros nombres comunes con los que se conoce a la especie son: huairacaspi, aguano o cedro mayna (Perú); achapo (Colombia); chuncho o seique (Ecuador), cedrorana, parica, lacaica o yacayaca (Brasil) (López, 1980-81; Encarnación, 1983; Richter \& Dallwitz, 2000).

Es un árbol grande, forma parte del estrato superior, fuste recto y cilíndrico, su altura total fluctúa entre 25 a $40 \mathrm{~m}$ y la comercial entre 15 a 25 $\mathrm{m}$. El diámetro a la altura del pecho es variable, encontrándose árboles de hasta $2 \mathrm{~m}$ de diámetro. Sus hojas son bipinnadas, alternas, con glándulas entre los pares de folíolos (Lao \& Flores, 1972; Lao, 1986; Spichiger et al., 1989). Las inflorescencias son terminales o axilares, con flores pequeñas, blanco cremosas (Lao \& Flores, 1972). El fruto tiene forma de cadena aplanada, al secarse adopta la forma de un tornillo, de 20-30 cm de longitud. Su semilla es plana, elíptica, de 3-3,5 $\mathrm{cm}$ de largo por $1,5 \mathrm{~cm}$ de ancho (Spichiger et al., 1989; López, 1980-81; Freitas et al., 1992).

La madera de esta especie tiene una densidad mediana, con duramen marrón claro, lustroso, mientras que la albura es más clara. La textura de la madera es gruesa, de grano recto a entrecruzado, fácil de trabajar con toda clase de herramientas de mano y máquinas, firme y resistente, con buen comportamiento al secado al aire libre (Aróstegui, 1978). La madera de tornillo es resistente al ataque de hongos descomponedores como Lenzites trabea, Polyporus sanguineus, Polyporus versicolor, Armillaria mellea y Ganoderma applanatum (Gonzáles \& Ames de Icochea, 1980-1981; Cardias \& Jesús, 1983), por lo que es utilizada en estructuras y carpintería de obras en general (Aróstegui, 1978; Loureiro \& Silva, 1968).

El tornillo crece de manera natural de 0 a $1500 \mathrm{~m}$, con climas que varían del subtropical al tropical y del seco al muy húmedo con rangos de temperatura que oscilan entre una máxima de $38^{\circ} \mathrm{C}$ y mínima de $23^{\circ} \mathrm{C}$. Soporta una alta precipitación, con amplitud de 2500 - $3800 \mathrm{~mm}$ de precipitación anual (López, 1980-81). La especie se asienta sobre una gran variedad de sustratos, entre los que predominan las arcillas caoliníticas y las arenas de naturaleza cuarzosa. En general, prefiere los suelos de buen drenaje, poco fértiles (López, 1980-81; Brako \& Zarucchi, 1993). Su distribución abarca la Amazonía, desde Perú hasta Brasil, Bolivia, Colombia, Ecuador, Surinam y Guyana Francesa (Maruyama, 1987; Boese, 1992; IBIF, 2007). En el
Perú, la especie se encuentra en los departamentos de Amazonas, Huánuco, Junín, Loreto, Madre de Dios, Pasco, San Martín, Ucayali y Cuzco (Encarnación, 1983; Brako \& Zarucchi, 1993).

La versatilidad de la madera de esta especie ha provocado que en los últimos años se haya extendido su extracción en los bosques naturales. En el Perú se comercializaron 1'792,802 $\mathrm{m}^{3}$ de madera rolliza de tornillo en el periodo 2003-2012, correspondiéndose esta cifra con más de cien mil árboles de $100 \mathrm{~cm}$ de DAP con altura comercial promedio de $20 \mathrm{~m}$. Sin embargo, la reposición del recurso no alcanzó ni el $10 \%$ del total extraído (Ministerio de Agricultura y Riego, 2013).

En la Amazonía peruana el tornillo constituye una de las especies forestales más experimentadas en condiciones de plantación, mostrando resultados alentadores en crecimiento en su estadio juvenil. A partir de esta información preliminar, se han masificado las plantaciones de esta especie, sin embargo, se conoce muy poco respecto a su crecimiento en periodos más extensos, situación que amerita la necesidad de investigar qué ocurre con esta especie en las etapas adultas de crecimiento.

Se ha priorizado la sistematización de los registros históricos sobre crecimiento de esta especie y el objetivo de este trabajo es desarrollar nuevos modelos de crecimiento y producción a partir de estos registros históricos de la especie, que tengan la suficiente exactitud en sus estimaciones como para aportar información útil para satisfacer la demanda de los gestores de bosques y contribuir con el aprovechamiento sostenible de la especie.

\section{MATERIAL Y MÉTODOS \\ Área de estudio}

Cedrelinga catenaeformis fue estudiada en parcelas permanentes de crecimiento (PPC), establecidas en los bosques de terraza alta en el ámbito del distrito de Jenaro Herrera (coordenadas UTM X -73.650777; Y -4.898929), localizado en el río Ucayali, en la selva baja de la Amazonía peruana. La descripción de las características de la terraza alta donde están ubicadas las plantaciones han sido detalladas por Claussi et al. (1992).

\section{Toma de datos}

Entre 1973 y 1998 se realizaron mediciones dasométricas multitemporales de 23 PPC instaladas en 13 plantaciones de tipo experimental silvicultural (1094 árboles), cuyas superficies varíaban de 0,063 a 1 ha. Cada PPC contaba entre 25 y 49 árboles y las densidades variaban entre 400 y 2500 pies/ha. Las 
mediciones incluyeron diámetro a la altura del pecho (DAP), altura total, altura comercial, proyección de la copa, tendencia del árbol y estado fitosanitario. El número de mediciones por plantación fue variable y se reportaron entre 4 y 9 mediciones realizadas en diferentes años.

Las plantaciones se establecieron en campo abierto con la finalidad de desarrollar técnicas silviculturales adecuadas para el manejo de Cedrelinga catenaeformis y fueron distribuidas en tres zonas experimentales denominadas campo abierto Laurent, El Piñal y El Cañal; todas sobre tierra firme.

A partir de los datos obtenidos en los inventarios de campo, se determinaron una serie de variables dendrométricas, tales como la sección normal y el volumen total del árbol, así como variables dasométricas, como la densidad $\left(N\right.$; pies ha $\left.{ }^{-1}\right)$, el área basimétrica del rodal $\left(G ; \mathrm{m}^{2}\right.$ ha $\left.{ }^{-1}\right)$, su volumen $\left(V ; \mathrm{m}^{3}\right.$ $\left.\mathrm{ha}^{-1}\right)$ y su altura dominante $(H ; \mathrm{m})$, definida como la altura media de los 100 pies más gruesos por hectárea y calculada como la altura media de los $k$ pies más gruesos de la parcela, siendo $k$ el entero inmediatamente superior al resultado de dividir la superficie de la parcela en $\mathrm{m}^{2}$ por 100 .

\section{Ajuste de las curvas de calidad de sitio para Cedrelinga catenaeformis}

Las curvas de calidad de sitio muestran la evolución de la altura dominante con la edad y son usadas, entre otras cosas, para predecir de forma indirecta la productividad del sitio. El índice de sitio se define como el valor de la altura dominante obtenido en estas curvas a una determinada edad de referencia y se suele emplear como indicador de calidad del sitio.

Las curvas de calidad del sitio deben cumplir una serie de propiedades, entre las que destacan las siguientes (Bailey \& Clutter, 1974; Cieszewski \& Bailey, 2000): polimórfismo, pauta de crecimiento sigmoide con un punto de inflexión, alcanzar una asíntota horizontal a edades avanzadas, tener un comportamiento lógico (por ejemplo, la altura dominante debe ser cero a la edad cero, la curva debe ser siempre creciente,...), ser invariantes con respecto al camino de simulación, es decir, que cuando se parte de la altura dominante $H_{1}$ a la edad $t_{1}$ y se estima con las curvas el valor de la altura dominante $H_{3}$ a la edad $t_{3}$ se debe obtener el mismo valor que si se estima primero la altura dominante $H_{2}$ a la edad $t_{2}$ y después se emplea este valor para estimar la altura dominante a la edad $t_{3}$; y por último, ser invariantes con respecto a la edad de referencia, es decir, la forma de las curvas no debe variar sea cual sea la edad de referencia que se emplee para definir el índice de sitio.

Para el ajuste de las curvas de calidad de sitio se empleó el método de las ecuaciones en diferencias algebraicas ADA, propuesto por Bailey \& Cluttter (1974). Se trata de un método invariante con respecto a la edad de referencia, que se basa en considerar que un parámetro del modelo base de crecimiento depende del sitio, para obtener finalmente una ecuación de la forma $Y_{2}=\mathrm{f}\left(Y_{1}, t_{1}, t_{2}\right)$.

Esta misma metodología de ecuaciones en diferencias algebraicas (ADA), ha sido empleada en este trabajo para ajustar las ecuaciones de evolución con el tiempo del diámetro, la altura y el volumen de los árboles individuales de Cedrelinga catenaeformis.

Existe un gran número de ecuaciones de crecimiento que se podrían emplear como modelos base para la construcción de las curvas de calidad de sitio (ver, por ejemplo las 74 ecuaciones documentadas por Kiviste et al., 2002). En este trabajo se han empleado tres funciones de crecimiento ampliamente usadas en este tipo de estudios: el modelo de Korf (1939), el modelo de Hossfeld (Hossfeld, 1822) y el modelo de Bertalanffy-Richards (Bertalanffy, 1949, 1957; Richards, 1959). Las expresiones matemáticas de estos modelos base figuran en la Tabla 1.

Para los ajustes de las ecuaciones en diferencias algebraicas, se emplearon todos los posibles pares de mediciones consecutivas de cada una de las variables analizadas. Los datos sobre crecimiento fueron ajustados en el paquete estadístico SAS/STAT (SAS Institute Inc., 2004) mediante mínimos cuadrados para modelos no lineales, empleando el método de iteración de GaussNewton. Puesto que los modelos empleados no son lineales, los valores inicializadores de la iteración se obtuvieron, en los casos en que fue posible, linealizando previamente la ecuación y realizando un ajuste por mínimos cuadrados ordinarios para modelos lineales. Cuando no fue posible la linealización, se usaron los valores obtenidos por otros autores en trabajos de similares características.

La bondad de ajuste de los modelos evaluados se ha basado en el análisis de dos estadísticos, obtenidos a partir de los residuos: la raíz del error medio cuadrático (REMC) y la eficacia del modelo (EM). Las expresiones matemáticas de los estadísticos antes comentados son las siguientes:

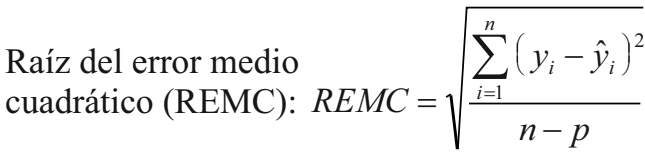




$$
\begin{aligned}
& \text { Eficacia del } \\
& \text { modelo }\left(\mathrm{Em}^{2}\right): E M^{2}=\left(1-\frac{\sum_{i=1}^{n}\left(y_{\mathrm{i}}-\hat{y}_{i}\right)^{2}}{\sum_{i=1}^{n}\left(y_{\mathrm{i}}-\bar{y}_{i}\right)^{2}}\right)
\end{aligned}
$$

Donde $y_{i}, \hat{y}_{i}$ e $\bar{y}_{i}$ son los valores observados, predichos y promedio, respectivamente, de la variable dependiente; $n$ es el número total de datos usados en el ajuste del modelo y $p$ es el número de parámetros a estimar.

Por último, para determinar la edad de referencia para definir el índice de sitio se tuvo en cuenta las siguientes consideraciones: 1) la edad base debe ser menor o igual que el turno de rotación silvicultural presentado bajo condiciones de manejo estándar de la plantación, b) la edad base debe estar próxima a la edad de rotación, $\mathrm{y}, \mathrm{c}$ ) la edad base debe ser tal que permita predecir de forma fiable la altura dominante a otras edades (Goelz \& Burk, 1992).

Teniendo en cuenta estas condiciones de partida, para determinar la edad de referencia ideal, se estimó el error relativo para diferentes valores de edades de referencia comprendidas entre 15 y 20 . Dicho error relativo se calcula con la siguiente expresión:

$$
R E \%=100 \frac{\sqrt{\sum_{i=1}^{n}\left(H_{i}-\hat{H}_{i}\right)^{2} /(n-p)}}{\bar{H}}
$$

Donde $H_{i}, \hat{H}_{i}, \bar{H}_{i}$ son los valores observados, predichos y promedio de altura dominante, respectivamente; $n$ es el número total de observaciones usadas en el modelo y $p$ es el número de parámetros en el modelo.

Los resultados fueron plasmados en un gráfico de tres entradas, donde se comparó el número de observaciones con el comportamiento del error relativo, la zona donde esta última variable se estabiliza, sugiere la mejor opción para establecerla como edad de referencia.

\section{RESULTADOS Y DISCUSIÓN}

\section{Estimación de la calidad de sitio y edad de referencia para Cedrelinga catenaeformis}

De todos los modelos ajustados a los datos observados, la ecuación en diferencias algebraicas (ADA) que deriva del modelo base de Korf (1939), en el que el parámetro " $b$ " depende del sitio (ecuación F8 de la tabla 1), fue la que obtuvo los mejores estadísticos de comparación. Por tanto, esta ecuación fue escogida para representar las curvas de calidad de sitio de las plantaciones de Cedrelinga catenaeformis estudiadas. La expresión matemática del modelo es la siguiente:

$$
H_{2}=e^{6,2403+\left(\ln H_{1}-6,2403\right) t_{1}^{0,4985} / t_{2}^{0,4985}}
$$

Donde $H_{2}$ y $H_{1}$ son los valores de la altura dominante a las edades $t_{2}$ y $t_{1}$, respectivamente y ln es el logaritmo neperiano. El modelo explicó el 95.45\% de la variabilidad observada en la altura dominante y el valor de la raíz del error medio cuadrático fue de $1.93 \mathrm{~m}$.

Una vez seleccionado este modelo, se han calculado los valores de los errores relativos en altura dominante para diferentes edades de referencia. En la Figura 1 se representan los valores de este error conjuntamente con el número de observaciones utilizadas en su cálculo. En la gráfica se puede observar que en el intervalo de 15-20 años (zona sombreada), los valores que representan a los errores relativos muestran cierta estabilidad con respecto al número de datos, por tal motivo, se consideró como adecuada una edad de referencia de 15 años para definir el índice de sitio. Esta edad coincide con la elegida por Otárola et al. (2001) para la misma especie. Además, esta edad de referencia es muy cercana al turno silvicultural recomendado para Cedrelinga catenaeformis (Baluarte \& Schwyzer, 2003). La Figura 1 también muestra que las edades tempranas no son muy recomendables para determinar el índice de sitio, debido al comportamiento errático de los valores correspondientes al error relativo en ese periodo de tiempo.

Una vez establecido el cálculo del índice de sitio a la edad de referencia de 15 años, se establecieron los rangos de clasificación para jerarquizar el índice de sitio por parcela en tres calidades, basándonos en los valores máximos y mínimos de las alturas dominantes observadas, del siguiente modo: las parcelas cuyo índice de sitio es mayor o igual que 25 $\mathrm{m}$ pertenecen a la clase de sitio alta; la clase de sitio media, corresponde a las parcelas cuya altura dominante se encuentra entre 21 y $24,99 \mathrm{~m}$ a la edad de referencia de 15 años, mientras que en la clase de sitio baja se encuentran las parcelas cuya altura dominante es inferior o igual de $20,99 \mathrm{~m}$.

En la Figura 2 se han representado las tres curvas limitantes de las calidades de sitio descritas, que corresponden a valores de índice de sitio de 19,25 y 31 metros de altura dominante a una edad de referencia de 15 años. En la misma figura se pueden observar las tendencias reales observadas en las parcelas utilizadas en los ajustes.

Según esta clasificación, cerca del $57 \%$ de las parcelas estudiadas corresponden a la clase de sitio alta; el $13 \%$ a la clase de sitio media y el $30 \%$ restante a la clase de sitio baja. 
Tabla 1. Modelos base y las ecuaciones en diferencias algebraicas que se derivan del despeje de los parámetros $a, b$ y c. Las ecuaciones en diferencias algebraicas fueron probadas para ajustar las curvas de calidad de sitio y los modelos de crecimiento en diámetro, altura y volumen de Cedrelinga catenaeformis. $Y_{1}$ e $Y_{2}$ son los valores de la variable dependiente a las edades $t_{1}$ y $t_{2}$ respectivamente; In es el logaritmo neperiano; $a, b y c$ son los parámetros a estimar.

\begin{tabular}{|c|c|c|}
\hline $\mathrm{N}^{0}$ & $\begin{array}{c}\text { Modelo base del cual al } \\
\text { despejar las variables a, b y c } \\
\text { se van a generar las Ecuaciones } \\
\text { de Diferencia Algebraica }\end{array}$ & $\begin{array}{l}\text { Ecuación en diferencia algebraica } \\
\text { que fueron usadas para ajustar las } \\
\text { curvas de calidad de sitio }\end{array}$ \\
\hline F1 & $\begin{array}{l}\text { Bertalanffy-Richards } \\
\qquad \begin{array}{l}Y=a\left(1-e^{-b t}\right)^{c} \\
\text { Despejando a }\end{array}\end{array}$ & $Y_{2}=Y_{1}\left(\frac{1-e^{-b t_{2}}}{1-e^{-b t_{1}}}\right)^{c}$ \\
\hline F2 & Despejando b & $Y_{2}=a\left(1-\left[1-\left(\frac{Y_{1}}{a}\right)^{1 / c}\right]^{t_{2} / t_{1}}\right)^{c}$ \\
\hline F3 & Despejando c & $Y_{2}=a\left(\frac{Y_{1}}{a}\right)^{\frac{\ln \left(1-e^{-b t_{2}}\right)}{\ln \left(1-e^{-b t_{1}}\right)}}$ \\
\hline F4 & $\begin{array}{c}\text { Hossfeld } \\
Y=t^{c} /\left(a+b t^{c}\right) \\
\text { Despejando a }\end{array}$ & $Y_{2}=\frac{t_{2}^{c}}{\frac{t_{1}^{c}}{Y_{1}}-b t_{1}^{c}+b t_{2}^{c}}$ \\
\hline F5 & Despejando b & $Y_{2}=\frac{t_{2}^{c}}{a+\left(\frac{t_{1}^{c} / Y_{1}-a}{t_{1}^{c}}\right) t_{2}^{c}}$ \\
\hline F6 & Despejando c & $Y_{2}=\frac{\frac{\ln \left(a / / / Y_{1}-b\right)}{\ln t_{1}}}{a+b t_{2}^{\frac{\ln \left(a / 1\left(Y_{1}-b\right)\right)}{\ln t_{1}}}}$ \\
\hline
\end{tabular}

Korf

F7 $\quad Y=e^{a-b / t^{c}}$

$$
Y_{2}=e^{\ln Y_{1}+b / t_{1}^{c}-b / t_{2}^{c}}
$$

Despejando a

F8

Despejando b

$$
Y_{2}=e^{a+\left(\ln Y_{1}-a\right) t_{1}^{c} / t_{2}^{c}}
$$

F9

Despejando c

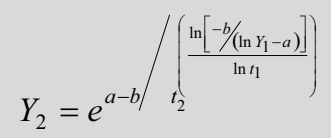




\section{Crecimiento en diámetro de árboles individuales de Cedrelinga catenaeformis}

La ecuación en diferencias algebraicas que mostró los mejores estadísticos de comparación, al ajustarla a los datos observados de crecimiento en diámetro, fue el modelo F3 (Tabla 1), derivado del modelo base de Bertalanffy-Richards (Bertalanffy, 1949, 1957; Richards, 1959), en la que el parámetro " $c$ " depende del sitio. Puesto que el crecimiento en diámetro está fuertemente condicionado por la calidad de sitio, se probaron diferentes expresiones que contenían el índice de sitio para caracterizar las asíntotas del crecimiento en diámetro (parámetro " $a$ " del modelo F3). Los mejores resultados se obtuvieron con la expresión potencial $b_{0} I S^{b_{1}}$, siendo $I S$ el índice de sitio y $b_{0}$ y $b_{l}$ parámetros a estimar en el ajuste. La ecuación en diferencias algebraicas finalmente obtenida para representar el crecimiento en diámetro de Cedrelinga catenaeformis en el área de estudio es la siguiente:

$$
d_{2}=29.5657 \cdot I S^{0.4844}\left(\frac{d_{1}}{29.5657 \cdot I S^{0.4844}}\right)^{\frac{\ln \left(1-e^{-0.035 / 2}\right)}{\ln \left(1-e^{-0.03511}\right)}}
$$

Donde $d_{2}$ y $d_{1}$ son los valores del diámetro $(\mathrm{cm})$ a las edades $t_{2} \mathrm{y} t_{1}$, respectivamente. El modelo explicó el $80,19 \%$ de la variabilidad observada en diámetros y el valor de la raíz del error medio cuadrático fue de $4,47 \mathrm{~cm}$.

En la Tabla 2 se muestran los valores de incremento corriente anual en diámetro para $C$. catenaeformis para los primeros años de desarrollo, calculados con la ecuación anterior para las tres condiciones de calidad de sitio definidas anteriormente (baja, media y alta).

En general, los más altos incrementos diamétricos son logrados en el primer año, con valores de 2,45, 2,63 y 2,77 para las clases de sitio bajo, medio y alto, respectivamente; en los siguientes años el incremento se ralentiza hasta cerca de $1 \mathrm{~cm}$, situación que ocurre en el año 7, 9 y 11 para las clases de sitio bajo, medio y alto, respectivamente. Los valores proyectados con la ecuación para la edad máxima de crecimiento registrado (27 años), son modestos, oscilando entre $27,69,30,20$ y $32,38 \mathrm{~cm}$ para las calidades baja, media y alta, respectivamente.

\section{Crecimiento en altura de árboles individuales de Cedrelinga catenaeformis}

La ecuación en diferencias algebraicas que mostró los mejores resultados en el ajuste de los datos de evolución de las alturas de los árboles individuales de Cedrelinga catenaeformis fue el modelo F8 (Tabla 1), que deriva del modelo base de Korf (1939), en el que el parámetro " $b$ " depende del sitio. Al igual que en el caso del crecimiento en diámetro, la asíntota del modelo (parámetro " $a$ ") fue considerado dependiente de la calidad de sitio, por lo que se ajustó la ecuación ADA, sustituyendo el valor de dicho parámetro con diferentes expresiones del índice de sitio (IS), obteniéndose los mejores resultados con la expresión $b_{0} I S^{b_{1}}$, siendo $b_{0}$ y $b_{1}$ parámetros a estimar en el ajuste. La expresión matemática de la ecuación en diferencias algebraicas finalmente obtenida fue la siguiente:

$$
h_{2}=e^{3.19 \cdot I S^{0,1138}+\left(\ln h_{1}-3.19 \cdot I S^{0.1138}\right) t_{1}^{0.5952} / t_{2}^{0.5952}}
$$

Donde $h_{2}$ y $h_{1}$ son los valores de altura (m) a las edades $t_{2}$ y $t_{1}$, respectivamente. El modelo explicó el $91,35 \%$ de la variabilidad observada en alturas y el valor de la raíz del error medio cuadrático fue de 2,32 $\mathrm{m}$.

Empleando esta ecuación se han estimado los crecimientos en altura para los tres escenarios de calidad de sitio antes definidos (baja, media y alta). Los valores de altura que se alcanzan a la edad de 27 años (máximo valor observado en los datos de parida), son de $21,52,24,75$ y $27,77 \mathrm{~m}$ para cada calidad de sitio, respectivamente.

Según este modelo de crecimiento, Cedrelinga catenaeformis también presenta crecimiento espectacular en altura los primeros años de su vida, donde alcanzan incrementos que fluctúan entre 3,09, 3,38 y $3,62 \mathrm{~m}$ en el primer año, hasta ralentizarse adquiriendo valores de 1,07; 1,08 y 1,09 a las edades de 6,7 y 8 años para las calidades de sitio bajo, medio y alto, respectivamente. Un rápido crecimiento en la etapa inicial es característico de especies con alto requerimiento de luz, confirmando el comportamiento heliófilo de esta especie (López, 1980-1981).

\section{Crecimiento en volumen de las plantaciones de Cedrelinga catenaeformis}

A partir de los datos de volumen de cada parcela analizada, en cada uno de los inventarios realizados, se ajustaron las ecuaciones en diferencias algebraicas de la Tabla 1, considerando que la asíntota de la ecuación depende de la calidad de sitio. Los mejores resultados de evolución del volumen con el tiempo, en plantaciones de Cedrelinga catenaeformis, se obtuvieron con el modelo F8, derivado del modelo base de Korf (1939), en el que el parámetro " $b$ " se considera dependiente del sitio y en el que la asíntota (parámetro " $a$ ") se relaciona con el índice de sitio como $b_{0} I S^{b_{1}}$, siendo $b_{0}$ y $b_{l}$ 
parámetros a estimar en el ajuste. La expresión matemática de la ecuación en diferencias algebraicas finalmente obtenida fue la siguiente:

$$
V_{2}=e^{1.4996 \cdot I S^{0.2751}+\left(\ln V_{1}-1.4996 \cdot I S^{0.2751}\right) t_{1}^{0.5818} / t_{2}^{0.5818}}
$$

Donde $V_{2}$ y $V_{1}$ son los valores de volumen $\left(\mathrm{m}^{3} \mathrm{ha}^{-1}\right)$ a las edades $t_{2}$ y $t_{1}$, respectivamente. El modelo explicó el $90.52 \%$ de la variabilidad observada en volúmenes de las parcelas y el valor de la raíz del error medio cuadrático fue de $35.38 \mathrm{~m}^{3}$ $\mathrm{ha}^{-1}$.

En la Figura 3 se muestran los valores observados de evolución de volumen en las plantaciones estudiadas así como las curvas ajustadas para los tres niveles de calidad de sitio definidos previamente: alto, medio y bajo.

Según esta función de crecimiento, Cedrelinga catenaeformis presenta también rápido crecimiento en volumen, alcanzando $360 \mathrm{~m}^{3} \mathrm{ha}^{-1}$ a la edad de 9 años en la clase de sitio alta, y $368 \mathrm{~m}^{3} \mathrm{ha}^{-1}$ a la edad de 18 años en la clase de sitio medio. Sin embargo, el incremento corriente anual - ICA en volumen concluye muy rápido, a la edad de 3 años alcanzando 36 y $56 \mathrm{~m}^{3} \mathrm{ha}^{-1}$ año ${ }^{-1}$ en las clases de sitio medio y alto respectivamente; mientras que en la clase de sitio bajo los árboles de Cedrelinga catenaeformis obtienen su máximo valor de ICA a la edad de 5 años con $16 \mathrm{~m}^{3} \mathrm{ha}^{-1}$ año ${ }^{-1}$. Con relación al incremento medio anual - IMA los valores más altos se producen a las edades de 5, 7 y 9 años, cuando los árboles de Cedrelinga catenaeformis alcanzan 44, 28 y $13 \mathrm{~m}^{3}$ $\mathrm{ha}^{-1} \mathrm{año}^{-1}$, en las clases de sitio alto, medio y bajo, respectivamente (Figura 4), y cuando los árboles cuentan diámetros de 14,16 y $17 \mathrm{~cm}$, respectivamente.

El aprovechamiento de los árboles de Cedrelinga catenaeformis, teniendo en cuenta los valores de IMA indicados, generaría una mayor producción de madera, sin embargo, los modestos diámetros alcanzados hasta esas edades constituyen una limitación fuerte para la cosecha, además de que la calidad de la madera no es la más recomendable (Baluarte, 2002). Desde el punto de vista del aprovechamiento industrial, es preferible esperar que los árboles cuenten con diámetros atractivos para el aserrío, lo que se alcanzaría, según el modelo de crecimiento en diámetro, a los 25 años, cuando los árboles alcancen DAP de 27, 29 y $32 \mathrm{~cm}$, para obtener entre 10,17 y $24 \mathrm{~m}^{3}$ ha $^{-1}$ año ${ }^{-1}$, en las clases de sitio baja, media y alta, cifra muy superior a lo que produce Cedrelinga catenaeformis en condiciones de bosque natural $\left(1 \mathrm{~m}^{3} \mathrm{ha}^{-1}\right.$ año $\left.^{-1}\right)$ (Schwyzer \& Bardales, 1982). Esta determinación tiene que tomarse a la luz de un riguroso estudio de costos que analice las diversas opciones planteadas a fin de tomar la decisión más apropiada.

Hay que tener en cuenta que los resultados comentados se corresponden con valores medios, que podrían variar en función de factores como la densidad de plantación. En todo caso, en este estudio se han analizado muy diferentes densidades de plantación (400 a 2500 pies/ha) y, por tanto, los resultados obtenidos son representativos de un amplio rango de densidades.

Tabla 2. Incrementos corrientes anuales en diámetro para Cedrelinga catenaeformis por clase de sitio bajo, medio y alto.

\begin{tabular}{|c|c|c|c|c|c|c|c|c|c|c|c|c|}
\hline \multirow{2}{*}{$\begin{array}{l}\text { Incremento corriente } \\
\text { anual }(\mathbf{c m})\end{array}$} & \multicolumn{12}{|c|}{ Edad (años) } \\
\hline & 1 & 2 & 3 & 4 & 5 & 6 & 7 & 8 & 9 & 10 & 11 & 12 \\
\hline Clase sitio bajo & 2.45 & 1.92 & 1.62 & 1.42 & 1.27 & 1.15 & 1.06 & 0.97 & 0.90 & 0.84 & 0.79 & 0.74 \\
\hline Clase sitio medio & 2.63 & 2.09 & 1.79 & 1.58 & 1.42 & 1.30 & 1.20 & 1.11 & 1.03 & 0.97 & 0.91 & 0.85 \\
\hline Clase sitio alto & 2.77 & 2.24 & 1.94 & 1.72 & 1.56 & 1.43 & 1.32 & 1.23 & 1.15 & 1.08 & 1.01 & 0.95 \\
\hline
\end{tabular}




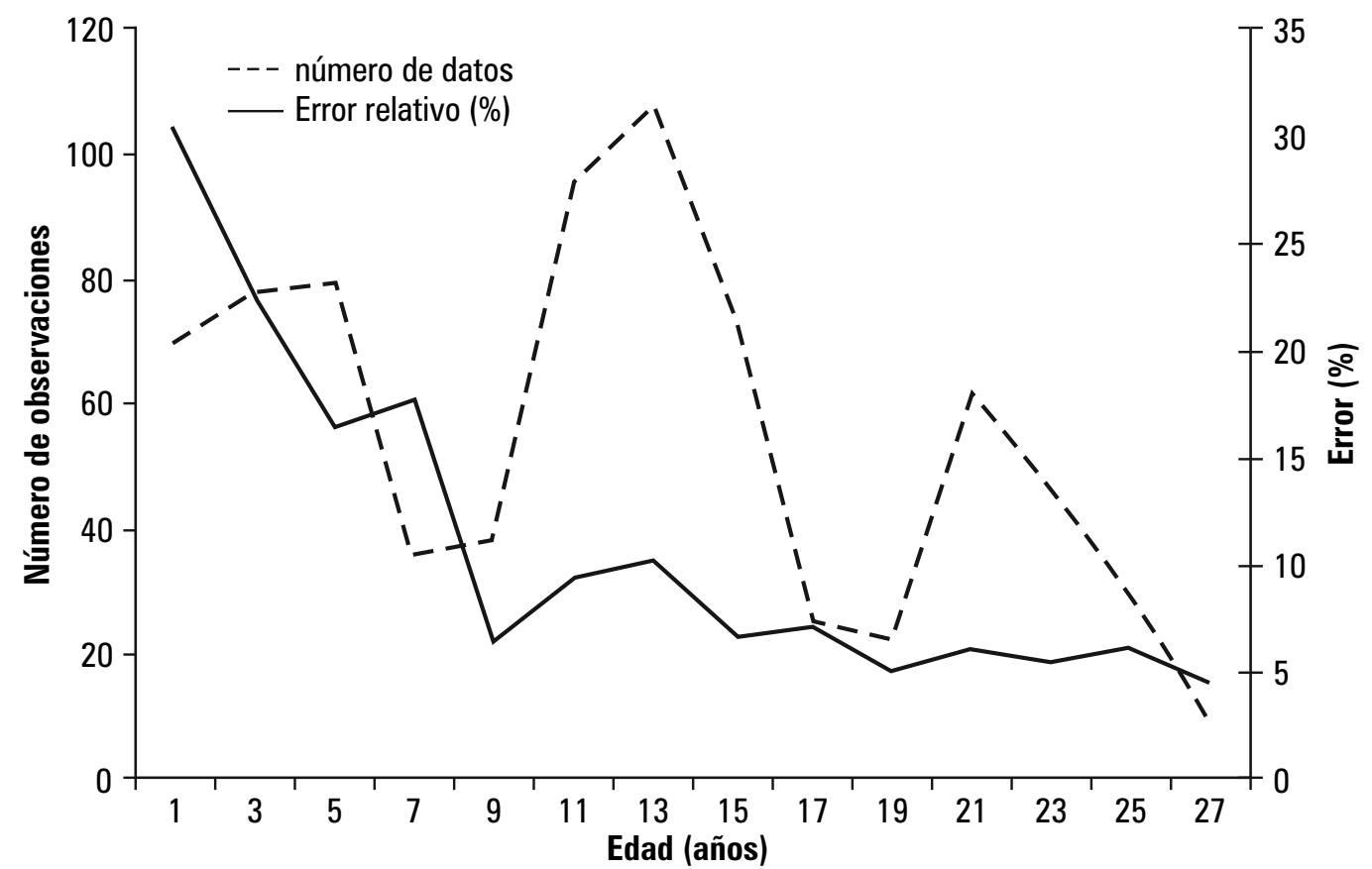

Figura 1. Valores del error relativo en la estimación de la altura dominante para diferentes valores de la edad de referencia obtenidos empleando el modelo (F8) derivado del modelo base de Korf. La zona sombreada representa los valores óptimos de edad de referencia teniendo en cuenta el error relativo obtenido y el número de datos empleado en su ajuste.

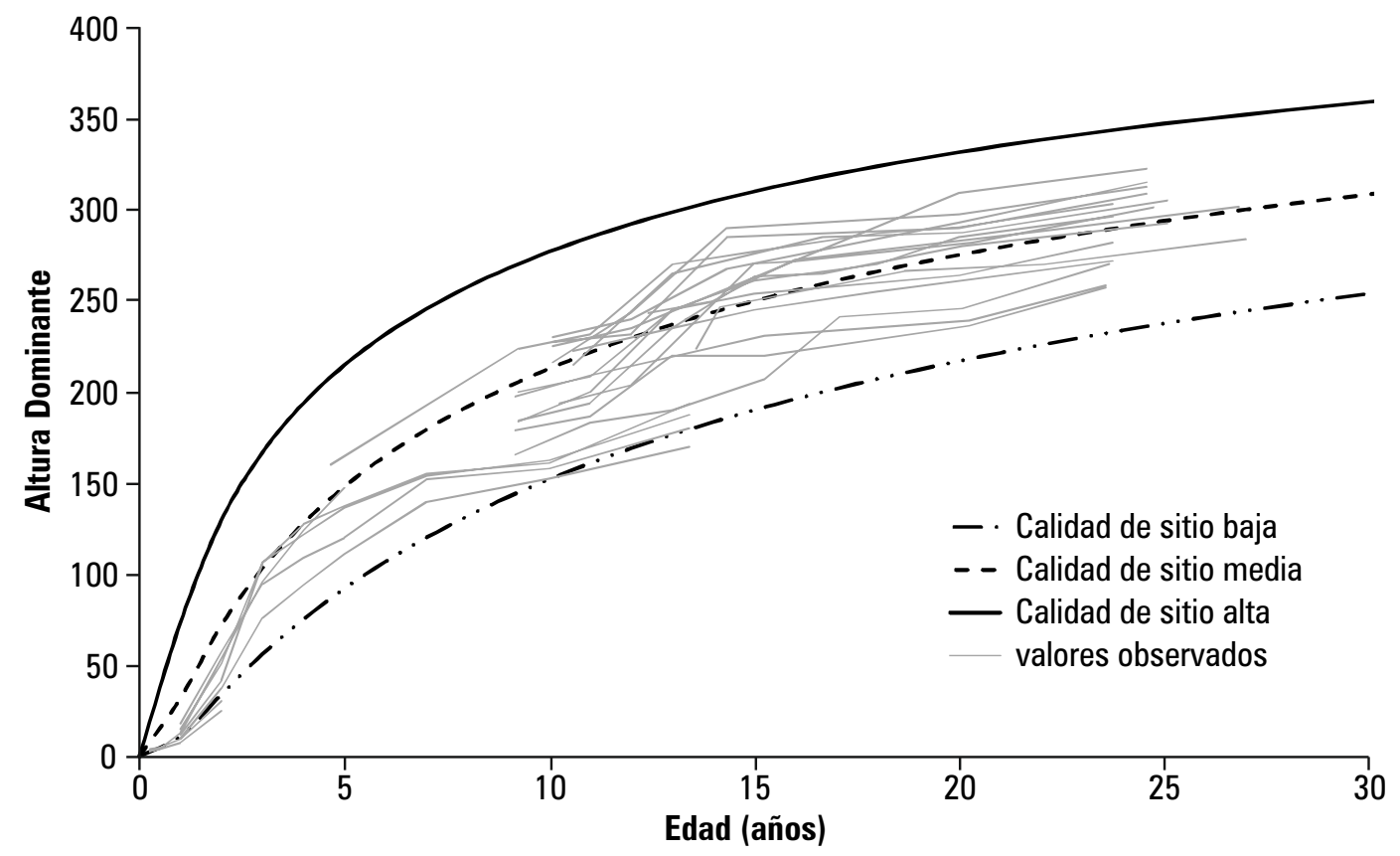

Figura 2. Modelo de crecimiento ajustado para estimar la calidad de sitio para Cedrelinga catenaeformis a la edad base de 15 años. 


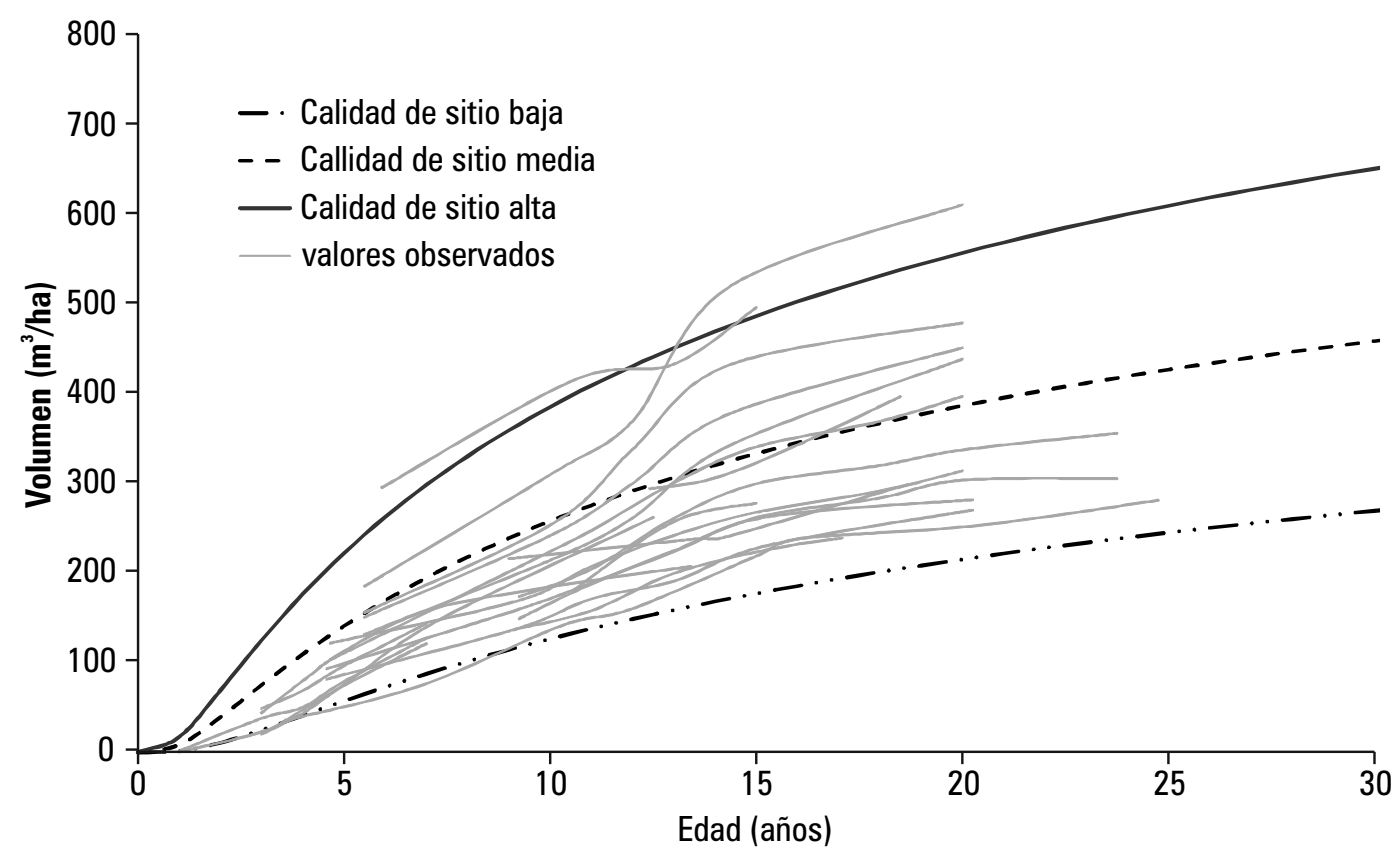

Figura 3. Curvas de crecimiento en volumen $\left(\mathrm{m}^{3}\right.$ ha $\left.\mathrm{A}^{-1}\right)$ para plantaciones de Cedrelinga catenaeformis por clases de sitio alto, medio y bajo, definidas por valores del índice de sitio de 19, 25 y 31 metros a la edad de referencia de 15 años.

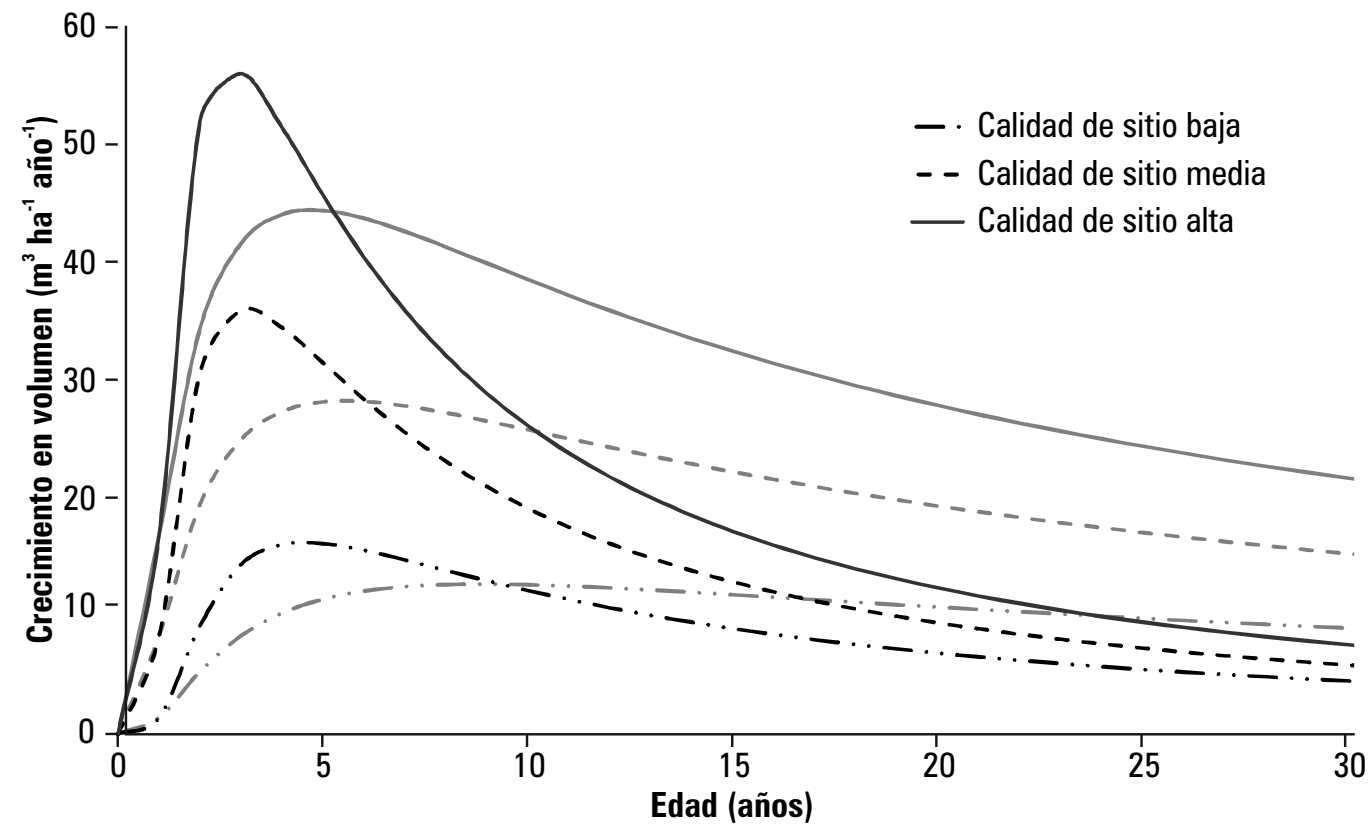

Figura 4. Incremento corriente anual e incremento medio anual en volumen para plantaciones de Cedrelinga catenaeformis en clase de sitio alto, medio y bajo, definidas por valores del índice de sitio de 19,25 y 31 metros a la edad de referencia de 15 años. 


\section{CONCLUSIONES}

La metodología empleada en la construcción del modelo de crecimiento para Cedrelinga catenaeformis ha mostrado un resultado aceptable de acuerdo con los estadísticos calculados que miden la bondad de ajuste del modelo, sin embargo, se trata de un modelo provisional y sería conveniente contar con un mayor volumen de datos, sobre todo en edades cercanas a la del turno de la especie, con el fin de poder reajustar el modelo abarcando un mayor periodo biológico de la especie para proporcionar una mayor fiabilidad al mismo.

\section{AGRADECIMIENTOS}

Queremos hacer un reconocimiento al Instituto de Investigaciones de la Amazonía Peruana - IIAP por facilitar los datos de campo de las parcelas permanentes de crecimiento de las plantaciones de tornillo del Centro de Investigaciones Jenaro Herrera, sin cuyo apoyo no habría sido posible generar este informe.

\section{REFERENCIAS BIBLIOGRÁFICAS}

Aróstegui, A. 1978. Estudio tecnológico de maderas del Perú (Zona Pucallpa). Vol I Características tecnológicas y usos de la madera de 145 especies del país. Ministerio de Agricultura, Universidad Nacional Agraria - La Molina. 483 pp.

Bailey, R.L.; Clutter, J.L. 1974. Base-age invariant polymorphic site curves. Forest Science 20: 155-159.

Baluarte, J. 2002. Implicancias silviculturales de los estudios tecnológicos de maderas de plantaciones de tres especies forestales del Centro de Investigaciones Jenaro Herrera Loreto. Instituto de Investigaciones de la Amazonía Peruana - IIAP. En: VIII Congreso Nacional Forestal y IV Asamblea Nacional del Capítulo de Ingenieros Forestales. Iquitos 6-8 Noviembre 2002. Perú. 15 pp.

Baluarte, J.; Schwyzer, A. 2003. Crecimiento y productividad de Cedrelinga catenaeformis Ducke "tornillo" en plantaciones. Instituto Nacional de Investigación Agraria - INIA. Informe de gestión 2003.19 pp.

Bertalanffy, L.v. 1949. Problems of organic growth. Nature 163: 156-158.

Bertalanffy, L.v. 1957. Quantitative laws in metabolism and growth. Quarterly Review of Biology 32: 217-231.

Boese, E. 1992. Actividades agroforestales y silviculturales en la región amazónica ecuatoriana. Red Agroforestal ecuatoriana. Quito, Ecuador. 138 pp.

Brako, L.; Zarucchi, J. 1993. Catálogo de las Angiospermas y Gimnospermas del Perú. Missouri Botanical Garden. Segunda reimpresión 1996. $1286 \mathrm{p}$

Cardias, M.F.C.; Jesús, M.A. 1983. Resisténcia natural de especies florestais da Amazónia ao ataque de fungos apodrecedores. Manaus, INPA. CPPF (Serie Técnica, 13).

Claussi, A.; Marmillod, D.; Blaser, J. 1992. Descripción silvicultural de las plantaciones forestales de Jenaro Herrera. Instituto de Investigaciones de la Amazonía Peruana. Iquitos, Perú. 334 pp.

Cieszewski, C.J.; Bailey, R.L. 2000. Generalized algebraic difference approach: theory based derivation of dynamic site equations with polymorphism and variable asymptotes. Forest Science 46: 116-126.

Encarnación, F. 1983. Nomenclatura de las especies forestales comunes en el Perú. Proyecto PNUD/FAO/PER/81/002. Documento de Trabajo $\mathrm{N}^{\circ}$ 7. Lima, Perú. 149 pp.

Freitas, M.; Medeiros, L.; de Lima, A. 1992. Leguminosas de la Amazónia brasileira - II Cedrelinga catenaeformis Ducke (Leg. Mimos.) Bot. Mus. Para. Emilio Goeldi, ser. Bot. 8(1):143-156.

Goelz, J.C.G.; Burk, T.E. 1992. Development of a well-behaved site index equation: jack pine in north central Ontario. Canadian Journal of Forest Research, 22:776-784.

Gonzáles, R.; Ames de Icochea, T. 1980-1981. Pudrición de la madera de diez especies forestales por acción de cinco hongos xilófagos. Universidad Nacional Agraria - La Molina, Ministerio de Agricultura. Revista Forestal del Perú V 10(1): 102-137.

Hossfeld, J.W. 1822. Mathematik für Forstmänner, Ökonomen und Cameralisten (Gotha, 4. Bd., S. 310).

Instituto Boliviano de Investigación Forestal - IBIF. 2007. Ecología y silvicultura de especies menos conocidas; mara macho, Cedrelinga catenaeformis (Ducke) LeguminosaeMimosaceae. Santa Cruz, Bolivia. 18 pp.

Kiviste, A.; Álvarez, J.G.; Rojo, A.; Ruiz, A. 2002. Funciones de crecimiento de aplicación en el ámbito forestal. Ministerio de Ciencia y Tecnología. Monografías INIA: Forestal No 4 . Madrid. 190 pp. 
Korf, V. 1939. Pfispevek k matematicke definici vzrustoveho zakona lesnich porostii. Lesnicka prace 18:339-356.

Lao, R. 1986. Descripción dendrológica de 51 especies forestales Asentamiento Rural Forestal von Humboldt. INADE, Proyecto Especial Pichis Palcazu. Documento mimeografiado. Huánuco, Perú. 52 p.

Lao, R.; Flores, S. 1972. Arboles del Perú; descripción de algunas especies forestales en Jenaro Herrera-Iquitos. 195 pp.

López, R. 1980-81. Estudio silvicultural del tornillo (Cedrelinga catenaeformis Ducke). Universidad Nacional Agraria - La Molina, Ministerio de Agricultura. Revista Forestal del Perú V 10(1):181-191.

Loureiro, A.; Silva, M. 1968. Catálogo das madeiras da Amazónia-I. Belem. SUDAM. p. 3-8.

Maruyama, E. 1987. Manejo de regeneración natural de tornillo (Cedrelinga catenaeformis Ducke) en la zona forestal de Alexander von Humboldt. Documento de trabajo $\mathrm{N}^{\circ}$ 03. INFOR COTESU. 39 pp.

Ministerio de Agricultura y Riego 2013. Perú Forestal en Números 2003-2012. Dirección General Forestal y de Fauna Silvestre. http//dgffs.minag.gob.pe/.Acceso: 03/11/2014

Otarola, E.; Freitas, L.; Linares, C.; Baluarte, J. 2001. Estimación de la calidad de sitio mediante "Índices de sitio" para Cedrelinga catenaeformis
Ducke (Tornillo) en plantaciones de Jenaro Herrera, Loreto (Perú). Instituto de Investigaciones de la Amazonía Peruana - IIAP. Folia Amazónica V12(1-2): 39-51.

Richards, F.J. 1959. A flexible growth function for empirical use. Journal of Experimental Botany 10 (29): 290-300.

Richter, H.G.; Dallwitz, M.J. 2000. Commercial timbers: descriptions, illustrations, identification, and information retrieval. Version: 25th June 2009. http://deltaintkey.com. Acceso: 19/08/2014

SAS Institute Inc., 2004. SAS/STAT ${ }^{\circledR} 9.1$ User's Guide. Cary, NC: SAS Institute Inc.

Schwyzer, A.; Bardales, F. 1982. El tornillo (Cedrelinga catenaeformis, Ducke). Proyecto de Asentamiento Rural Integral Jenaro Herrera. Iquitos, Perú. 33 pp.

Spichiger, R.; Meroz, J.; Loizeau, P.; Stutz de Ortega, L. 1989. Contribución a la flora de la Amazonía peruana; los árboles del arborétum Jenaro Herrera. V 1. Conservatorio y Jardín Botánicos de la ciudad de Ginebra, Organización Suiza para el Desarrollo y la Cooperación, Instituto de Investigaciones de la Amazonía Peruana. Ginebra, Suiza. 359 pp.

Recibido: 10 de febrero del 2015

Aceptado para publicación: 14 de abril del 2015 
